\title{
Antimicrobial Prophylaxis for Women with First Trimester Vaginal Bleeding Could Reduce the Rate of Miscarriage
}

\author{
Kaima Abdullah Frass ${ }^{1}$, Amat-Al Karem Ali Al Huri ${ }^{1}$, Abdelrahman Hasan Al Harazi ${ }^{2, *}$ \\ ${ }^{1}$ Department of Obstetrics and Gynecology, Faculty of Medicine, Sana'a University, Sana'a, Yemen \\ ${ }^{2}$ Department of Obstetrics and Gynecology, Faculty of Medicine, Thamar University, Sana'a, Yemen
}

\section{Email address:}

KaimaFrass@hotmail.com (Kaima A. F.), osama.m.alshami@gmail.com (Amat-Al K. A. Al H.), yem008@yahoo.com (Abdelrahman H. Al H.)

${ }^{*}$ Corresponding author

To cite this article:

Kaima Abdullah Frass, Amat-Al Karem Ali Al Huri, Abdelrahman Hasan Al Harazi. Antimicrobial Prophylaxis for Women with First Trimester Vaginal Bleeding Could Reduce the Rate of Miscarriage. Journal of Gynecology and Obstetrics. Vol. 5, No. 3, 2017, pp. 42-45. doi: $10.11648 /$ j.jgo.20170503.12

Received: May 3, 2017; Accepted: May 11, 2017; Published: June 28, 2017

\begin{abstract}
This is a prospective study conducted to examine the possible benefit of antibiotic prophylaxis in reducing the symptoms of threatened miscarriage as well as decreasing the rate of progression into spontaneous miscarriage in women with first trimester vaginal bleeding. This study was conducted at Al Thawra General Hospital over a year (from Jan. to Dec 2016). Ninety-two women with threatened miscarriage during the first trimester were included (case group) and compared to 90 pregnant women without vaginal bleeding with similar parity and gestational age. Amoxicillin-clavulanate drug was given for all cases of the study group during vaginal bleeding. Both groups were monitored up to 28 weeks gestational age. Among 92 women with threatened miscarriage, only three cases $(3.2 \%)$ had spontaneous loss $(\mathrm{P}<0.001)$. Vaginal bleeding was significantly relieved within 3 days of antibiotic administration $(P<0.00)$. Results of this study give evidence that antibiotic prophylaxis for women with threatened miscarriage reduces the amount and duration of vaginal bleeding, and decreases the rate of progression into spontaneous miscarriage.
\end{abstract}

Keywords: Women, First-Trimester Bleeding, Miscarriage, Antibiotic

\section{Introduction}

First - trimester vaginal bleeding is a common complication that affects $16-25 \%$ of all pregnancies in women [1]. Miscarriage is defined as pregnancy termination prior to 20 weeks of gestation or a fetus born weighing less than $500 \mathrm{~g}$. The clinical diagnosis of threatened miscarriage is presumed when a vaginal bleeding appears through a closed cervix during the first half of pregnancy [2]. The usual presentation is slight vaginal bleeding or spotting following a period of amenorrhea. Pain is often minimal, and in the form of pelvic ache or low backache [3].

A definitive diagnosis of threatened miscarriage is frequently made following ultrasonographic examination confirming the presence of fetal heart activity in an intrauterine pregnancy [4]. About one fourth of all pregnant women experience spotting or bleeding in the first several weeks of pregnancy and one half of those who bleed will miscarry [5].

As the bleeding in most cases of threatened miscarriage originates from placenta, there is an increased-risk of adverse pregnancy outcome related to placental dysfunction such as spontaneous miscarriage, preeclampsia, premature rupture of membranes(PROM), intrauterine growth restriction (IUGR), preterm labor, and placental abruption[6]. The causes of threatened miscarriage are unknown and therefore, the optimal management are still unclear and nonspecific.

The role of the cervicovaginal infections in causing spontaneous miscarriage has been documented [7]. Although it is reported that the prevalence of Chlamydia trachomatis infection of the female genital tract has dramatically increased over the past 10 years, its role in miscarriage is still unclear [8]. This could possibly be related to the fact that more than $80 \%$ of cases are asymptomatic and thus unrecognized. Another study reported that the pregnant 
women are susceptible to Listeria Monocytogenes infection which can be asymptomatic or can give rise to subclinical symptoms. This infection invades the placenta resulting in miscarriage, preterm birth, and stillbirth among others [9].There is a very limited data in the literature pertaining to the usefulness of antibiotics prophylaxis in the threatened miscarriage. Up to the best of our knowledge, only one study was conducted retrospectively to test the usefulness of antibiotics in women with threatened abortion and concluded the usefulness of early antibiotic therapy in preventing pregnancy loss in the first trimester [9].

We hypothesized that, threatened miscarriage might be triggered by certain asymptomatic infectious process within the genital tract therefore, broad-spectrum antibiotic could eliminate such process and thus shorten the duration of symptoms.

The purpose of this study was to examine the efficacy of antibiotic prophylaxis in reducing the symptoms of threatened miscarriage as well as decreasing the rate of progression into spontaneous miscarriage for women with first trimester vaginal bleeding.

\section{Methods}

This is a prospective study conducted at Al Thawra General Hospital over a year (from Jan. $1^{\text {st }}$ to Dec $311^{\text {st }}$ 2016).The study was performed in accordance to the Helsinki declaration. The ethics committee of the hospital approved the study and a written consent was obtained from all subjects after full explanation of the study purpose. The inclusion criteria were singleton pregnancy up to 14 weeks with positive fetal heart activity seen by ultrasound, vaginal bleeding in the presence of closed and uneffaced cervix, negative results of infection screening, no medical disorder or known cause of miscarriage, and they have no allergy for amoxicillin-clavulanate drug. We excluded from this study any women with an opened cervix $(\geq 2 \mathrm{~cm})$, previous 2 or more miscarriages, or when the bleeding is caused by local factor such as cervicitis, cervical lesions, cervical polyps, trauma or caused by systemic disorders.

Ninety two women met our criteria and included in this study. For the purpose of comparison, another group of 90 women without vaginal bleeding was chosen as a control. The control group was selected by $1: 1$ ratio as for each patient of the study group, the next woman attended the clinic for early pregnancy check-up, comparable with age, parity, and gestational age was chosen as a control, given that all women were available for follow-up. No intervention was made for the control group aside from routine pregnancy check -up.

All women of the study group underwent detailed history, physical examination, and investigations. The history included maternal age, parity, last menstrual period (LMP), regularity of the menstrual cycles, previous pregnancy history and medication use. Exploration of the presenting complaint including the onset of the bleeding, amount, duration, colour and consistency, symptoms like pain, cramping and vomiting were noted. Physical examination was performed and included a vaginal speculum examination for identifying the amount and characteristics of bleeding and visualization of the cervix and vagina.

The investigations requested were complete blood count with differential, blood typing with $\mathrm{Rh}$ identification, quantification of $\beta$-hCG and screening for infection if indicated. Screening for women suspected as having infection was carried out using the suitable testing such as high vaginal swab (HVS), urinalysis, urine culture and sensitivity, and blood culture as indicated by history and physical examination. A detailed transabdominal or transvaginal ultrasound was performed as appropriate to confirm the viability of the fetus. Bleeding was considered slight when spotting, and heavy when the amount of bleeding equal or greater than that of the menstrual cycle.

Both groups were similarly followed-up to the viability of fetus. Women of the study group were treated in the hospital clinic as outpatients and given Augmentin (amoxicillinclavulanate; Smith Kline Beecham; Philadelphia, PA) 2 g initially, then $1 \mathrm{~g}$ each 12 hours orally for 7 days (commenced at times of bleeding and at different gestational age). Folate (Alpha Pharmaceutical, India) supplementation was prescribed as $4 \mathrm{mg}$ daily for all women who did not receive it before. They were advised for bed rest and abstaining from coitus during the treatment course. They were instructed to return to the clinic for follow-up one week after drug taking and 4 weekly thereafter up to 28 weeks of gestation. In each visit, the patient's symptoms were evaluated along with complete antenatal check-up including bedside ultrasound as appropriate. We defined the success of antibiotic in arresting the process of threatened miscarriage as cessation of vaginal bleeding with the presence of living fetus seen by ultrasound at 28 weeks of gestation.

Statistical analysis

Analysis of the data was carried out using SPSS version 21. The values given are mean $\pm \mathrm{SD}$ or percentages as appropriate. Independent sample t-test was used to evaluate the association between continuous variables and Chi-square test for categorical variables. Odds ratio and $95 \%$ confidence interval were calculated as appropriate. A p value of $\leq 0.05$ was considered statistically significant.

\section{Results}

There were 92 cases diagnosed as having threatened miscarriage and 90 cases were chosen as a control group. They did not have any significant differences in age, parity, or gestational age. The maternal characteristics are summarized in table 1 . Bleeding before 9 weeks of gestation was more prevalent than that after 9 weeks $(68.4 \%$ vs. $31.5 \%)$. The difference was statistically significant $(P<0.001)$. The slight bleeding was noted among $53.2 \%$ versus $46.7 \%$ who had heavy bleeding.

Vaginal bleeding and the associated lower abdominal pain were relieved within 3 days after amoxicillin-clavulanate medication in $83.7 \%$, while the remaining $15.3 \%$ were 
completely relieved within 7 days of the therapy initiation. The incidence of spontaneous miscarriage among the treated group was three cases (3.2\%) and almost 96.7\% had continued their pregnancies beyond 28 weeks gestation compared to $(3.3 \%)$ of the control group. The difference between the two groups was insignificant ( $p$ 0.69). Of these three miscarriages, two cases occurred among women who had heavy vaginal bleeding and the third one occurred among women with slight bleeding. Table 2 .

Table 1. Maternal characteristics of the study and control groups.

\begin{tabular}{llll}
\hline Variable & case group & control group & P value \\
\hline & $(\mathbf{n = 9 2 )}$ & $\mathbf{( n = 9 0 )}$ & \\
\hline Age (yr) & $27.98 \pm 7.9$ & $27.47 \pm 7.6$ & 0.65 \\
Parity & & & \\
Primigravida & $21(22.8)$ & $25(27.8)$ & 0.21 \\
Multigravida & $71(77.17)$ & $65(72.2)$ & 0.22 \\
Residence & & & \\
Rural & $23(25)$ & $26(28.9)$ & 0.32 \\
Urban & $69(75)$ & $64(71.1)$ & 0.27 \\
Education & & & \\
None & $12(13)$ & $11(12.2)$ & 0.41 \\
Primary & $33(35.9)$ & $28(31.1)$ & 0.24 \\
Secondary & $34(36.9)$ & $40(44.4)$ & 0.15 \\
High & $13(14.1)$ & $11(12.2)$ & 0.34 \\
Socioeconomic & & & \\
Low & $14(15.2)$ & $13(14.4)$ & 0.41 \\
Medium & $55(59.8)$ & $58(64.4)$ & 0.33 \\
High & $23(25)$ & $19(21.1)$ & 0.26 \\
History of one miscarriage & $7(7.6)$ & $5(5.5)$ & 0.00 \\
History of previous PTL & $9(9.8)$ & $6(6.6)$ & 0.00 \\
\hline
\end{tabular}

The data are presented as mean $\pm \mathrm{SD}$ or $\mathrm{n}(\%)$.

$\mathrm{PTL}=$ preterm labor.

Table 2. The study outcome.

\begin{tabular}{|c|c|c|c|}
\hline & Study group & control group & \\
\hline & $(n=92)$ & $(n=90)$ & $P$ value \\
\hline \multicolumn{4}{|l|}{$\begin{array}{l}\text { Gestational age,wk } \\
\text { (at bleeding) }\end{array}$} \\
\hline$<9$ & $63(68.4)$ & $64(71.1)$ & \\
\hline$>9$ & $29(31.5)$ & $26(28.9)$ & 0.69 \\
\hline \multicolumn{4}{|c|}{ Estimated vaginal bleeding } \\
\hline Slight & $49(53.2)$ & ---- & \\
\hline Heavy & $43(46.7)$ & & \\
\hline \multicolumn{4}{|c|}{ Duration of symptoms, days } \\
\hline $1-3$ & $77(83.7)$ & ---- & \\
\hline $4-7$ & $15(15.3)$ & & \\
\hline Spont. miscarriage & $3(3.2)$ & $3(3.3)$ & 0.69 \\
\hline
\end{tabular}

The data are presented as mean $\pm \mathrm{SD}$ or $\mathrm{n}(\%)$.

\section{Discussion}

The current study found that the symptoms of threatened miscarriage were settled in the majority of patients $(83.7 \%)$ within the first 3 days of amoxicillin-clavulanate administration $(P<0.001)$.In addition, it is observed that the rate of spontaneous miscarriage among women with threatened miscarriage was lower than that reported by other studies[5]. These findings suggest that a focus of local infection may have a causative role.

One of the most parameter used to estimate fetal loss during the first trimester is the presence of fetal heart activity by transvaginal ultrasound after 10 weeks of gestation. When demonstrated, the fetal loss rate has been estimated to be 2$5 \%$ for women 35 years or younger versus $16 \%$ in women older than 35 years [2]. However, the loss rate after threatened miscarriage may be higher [11]. The incidence of fetal loss reported varies among studies due to the fact that many studies are retrospective, of small sample size or relied on patients recall recruiting later in pregnancy. Agrawal $\mathrm{S}$ and colleagues (2014) in a small prospective case control study found the overall spontaneous miscarriage rate for patients after diagnosis of a viable pregnancy by ultrasound as $21 \%$ for cases versus $5.45 \%$ for control group [12].

In the current study because the presence of fetal cardiac activity was an essential part of the inclusion criteria, the overall loss rate was $3.2 \%$. This favorable outcome most likely resulted from infection treatment and the rate of spontaneous loss could be reduced by antibiotic therapy, similar to the finding of another study [9]. Our long experience in the management of miscarriage have been shown that the majority of the histopathology studies of the products of conception often reveal infected products which suggests that the infection is one of the primary causes of miscarriage. During pregnancy genital mucosa becomes thinner and has greater surface area, making pregnant women more susceptible to infection [13]. Furthermore, it is reported that infection such as chlamydia trachomatis is an important causative factor of miscarriage and the association between chlamydia positive diagnostic test and miscarriage is significant (OR 2.41,95\% CI 1.32-3.35, $P<0.01$ ) [8].

Weiss JL et al in a large study found, among 16506 patients that the first trimester vaginal bleeding is associated with a significant increased risks of preeclampsia, preterm labor, PROM, and placental abruption [1].Another study found a significant increased of the rate of placental abruption, but preeclampsia and small for gestational age were not significantly associated [14-16].

A strength of this study is that all subjects were collected and managed at the time of vaginal bleeding therefore, the likelihood of recall bias is low. Another strength is that all women were followed up regularly till fetal viability with no drop out. In addition, the selection of amoxicillin-clavulanate is based on the knowledge that it could not add concern of the possible teratogenic effect on the developing fetusand it is associated with less frequent nausea and vomiting which may be already existed in some women during the first trimester. The limitation of this study is that the amount of bleeding was estimated subjectively as described by patients.

We suggest a future studies to extend amoxicillinclavulanate prophylaxis beyond the first trimester as 5-7 days in each calendar month till birth. These proposed studies should assess if such extended antibiotic prophylaxis could lower the rate of adverse pregnancy outcome particularly preterm labor. 


\section{Conclusion}

Our study gives evidence that amoxicillin-clavulanate prophylaxis for women with threatened miscarriage has a favourable prognostic outcome in terms of reducing the amount and duration of vaginal bleeding, relieving the associated symptoms and decreasing the rate of progression into spontaneous miscarriage. Antibiotic can be used to help patients who develop first trimester vaginal bleeding to minimize the chance of miscarriage.

\section{References}

[1] Weiss JL, Malone FD, Vidaver J et al. Threatened miscarriage: A risk factor for poor pregnancy outcome, a population-based screening study. Am J Obstet Gynecol. 2004 Mar; 190(3):745750.

[2] Maged AM, AI Mostafa M. Biochemical and ultrasonographic predictors of outcome in threatened miscarriage. Middle East Fertility Society Journal 2013; 18, 177-181.

[3] Tamizian O, Arulkumaran S. Bleeding in early pregnancy. Current Obstetrics \& Gynaecology 2004; 14, 23-33.

[4] Davari-Tanha F, Shariat M, Kaveh M, Ebrahimi M, Jalalvand S. Threatened miscarriage: a risk factor for poor pregnancy outcome. Acta Medica Iranica. 2008; 46(4):314-20.

[5] Paspulati RM, Bhatt S, Nour SG. Sonographic evaluation of first trimester bleeding. Radiol Clin North Am. 2008; $46(2): 437$.

[6] Wijesiriwardana, A., Bhattacharya, S., Shetty, A., et al. Obstetric outcome in women with threatened mis-carriage in the first trimester. Obstetrics \& Gynecology, 2006; 107, 557562 .

[7] Tibaldi C, Cappello N,. Latino MA, Polarolo G, Masuelli G,
Cavallo F, et al. Maternal risk factors for abnormal vaginal flora during pregnancy. International Journal of Gynecology and Obstetrics 2016; 133: 89-93.

[8] Visnovsky J, Biskupska-Bodova K, Cabanova B, Kudela E, Dokus K. Early Fetal Loss and Chlamydia Trachomatis Infection. GynecolObstet2013; 3(5):3-5.

[9] Ou MC, Pang CC, Chen FM, Su CH, Ou D. Antibiotic treatment for threatened abortion during the early first trimester in women with previous spontaneous abortion. Acta Obstet Gynecol Scand. 2001; 80:753-756. [PubMed].

[10] Jamshidi M, Jahromi1AS, Davoodian P, Amirian M, Zangeneh $\mathrm{M}$, JadcarehF et al. Seropositivity for listeria monocytogenes in women with spontaneous abortion: A case-control study in Iran. Taiwan J ObstetGynecol• 2009; 48 (1):46-48.

[11] Tannirandorn Y, Sangsawang S, Manotaya S et al. Fetal loss in threatened miscarriage after embryonic/ fetal heart activity. Int J GynaecolObstet 2003; 81:263-6.

[12] Agrawal S, Khoiwal S, Jayant K, Agarwal R. Predicting adverse maternal and perinatal outcome after threatened miscarriage. Open Journal of Obstetrics and Gynecology. 2014; 4(1):1-7.

[13] Fonseca TM, Cesar JA, Mendoza-Sassi RA, Schmidt EB. Pathological vaginal discharge among pregnant women: pattern of occurrence and association in a population-based survey. ObstetGynecol Int. 2013; 2013:590416.

[14] Dadkhah F, Kashanian M, Eliasi G. A comparison between the pregnancy outcome in women both with or without threatened miscarriage. Early Human Development 2010;86: 193-196.

[15] Johns J, Jauniaux E. Threatened miscarriage as a predictor of obstetric outcome.ObstetGynecol2006; 107(4):845-50.

[16] Calleja-Agius JC, Calleja N, Brincat M, Dorianne Spiteri D. Obstetric outcome in cases of threatened spontaneous miscarriage. Int J Gynaecol Obstet. 2010 Jul; 110(1):75-6. 